Original Paper http://ajol.info/index.php/ijbcs http://indexmedicus.afro.who.int

\title{
Evaluation des connaissances et de l'impact des pratiques des populations riveraines sur la conservation de l'avifaune de la Forêt de Burhinyi (Itombwe, Sud-Kivu, RD Congo)
}

\author{
Bertin Cisirika MURHABALE ${ }^{1 *}$, Blaise Cimira IRENGE ${ }^{1}$, Gabriel Kadahanwa \\ BIRINGANINE ${ }^{2}$, Frank A. BAPEAMONI ${ }^{3}$, Charles M. KAHINDO ${ }^{1}$ et \\ Dieudonné A. UPOKI ${ }^{3}$
}

\author{
${ }^{1}$ Université Officielle de Bukavu, Faculté des Sciences et Sciences Appliquées, Département de Biologie, \\ B.P.570, Bukavu, République Démocratique du Congo. \\ ${ }^{2}$ Institut Supérieur des Techniques Appliquées de Bukavu à Butuza, République Démocratique du Congo \\ ${ }^{3}$ Université de Kisangani, Faculté des Sciences, Département de Biologie, Laboratoire d'Ecologie et Gestion \\ des Ressources Animales, B.P. 2012, Kisangani, République Démocratique du Congo \\ *Auteur correspondant ; E-mail : bertinmurhabale@gmail.com; Tel : +243998857787
}

\section{RESUME}

A l'instar de nombreuses autres forêts des montagnes non protégées, la forêt de Burhinyi, faisant partie du massif d'Itombwe, subit une forte pression due aux activités humaines qui impactent négativement la valeur globale du site. Nous avons évalué les connaissances et l'impact de certaines pratiques des populations riveraines de la forêt de Burhinyi sur la conservation de l'avifaune en vue d'obtenir les données de base susceptibles d'orienter les initiatives de conservation communautaire en cours. Un questionnaire d'enquête a été utilisé pour collecter les données auprès de 188 personnes issues des différents ménages choisis dans 6 villages. Cette étude révèle que les populations riveraines exercent un bon nombre d'activités ayant un effet néfaste sur la biodiversité forestière. Il s'agit notamment des techniques de chasse, des pratiques agricoles et de l'exploitation minière. Sur 22 taxons couramment abattus à Burhinyi, une moyenne de 4,6 oiseaux est tuée journalièrement. Six taxa (Aigles, Hiboux, Corbeaux, Buses montagnardes, Gonoleks et Touracos) sont exploités en médecine traditionnelle et comme ornement des tenues portées par les chefs locaux pendant les cérémonies coutumières. Les connaissances des populations riveraines sur l'avifaune sont larges. Toutefois, elles sont significativement variables selon le groupement d'origine mais pas selon le niveau d'études. L'importance de la conservation des oiseaux a été reconnue par $80,1 \%$ des enquêtés contre $14,5 \%$ qui s'y opposent et $0,5 \%$ d'indécis.

(C) 2020 International Formulae Group. All rights reserved.

Mots clés : Perception, communautés, protection, oiseaux, Rift Albertin.

\section{Assessment of knowledge and impact of practices by riparian populations of Burhinyi Forest (Itombwe, South-Kivu, DR Congo) on the conservation of avifauna}

\section{ABSTRACT}

Like many other unprotected mountain forests, the Burhinyi forest is under great pressure due to human activities, which negatively impact the global value of the site. We assessed the knowledge and impact of some practices within riparian populations around Burhinyi forest on the conservation of avifauna to obtain baseline 
data that could guide ongoing community conservation initiatives. A survey questionnaire was used to collect data from 188 people from households selected in 6 villages. This study shows that riparian populations are engaged in a number of activities that have a negative impact on forest biodiversity. These include hunting techniques, agricultural practices and mining. Of the 22 taxa commonly trapped in Burhinyi, an average of 4.6 birds are killed daily. Six taxa (Eagles, Owls, Ravens, Mountain Hawks, Gonoleks and Touracos) are used in traditional medicine and sources of ornaments of attires worn by local leaders during customary ceremonies. The knowledge of the riparian populations about the avifauna is broad. However, they vary significantly by area of origin and not by education level. The importance of bird conservation was recognized by $80.1 \%$ of respondents compared with $14.5 \%$ who opposed it and $0.5 \%$ who were undecided.

(C) 2020 International Formulae Group. All rights reserved.

Keywords : Perception, communities, protection, birds, Albertine Rift.

\section{INTRODUCTION}

La biodiversité est confrontée à une grave crise d'extinction avec des pertes d'espèces qui atteignent des niveaux trois fois plus importants que les moyennes estimées sur les temps géologiques (Claver et al., 2011 ; Munay, 2004 ; Brooks et al., 2007).

Globalement, les activités humaines réduisent la diversité des espèces, les structures des peuplements et des processus écologiques à diverses échelles spatiales (Olden et al., 2004 ; Ladle et Whittaker, 2011 ; Chapin et al., 2000 ; Fahrig, 2003 ; Foley et al., 2005). La perte de l'habitat à travers l'agriculture (Tscharntke et al., 2005 ; Tilman et al., 2002), la chasse et l'exploitation des ressources naturelles ont déterminé l'appauvrissement spécifique et quantitatif de la flore et de la faune sauvages, ce qui est le cas particulièrement des oiseaux. La destruction accélérée des forêts tropicales fait disparaître d'irrémédiable manière les espèces qui leur sont intimement liées. C'est parmi elles que l'on compte les oiseaux les plus menacés, certains se trouvant même au bord de l'extinction (Barnosky et al., 2011).

Certaines espèces d'oiseaux sont aujourd'hui rares ou menacées ; cela est essentiellement dû à diverses pressions anthropiques comme la chasse, la réduction des surfaces des écosystèmes, l'utilisation des pesticides (insecticides et herbicides), la demande croissante d'espace d'habitation autour des grandes villes (Lougbégnon et Libois, 2011). Les prélèvements sur l'avifaune qui se réalisent pour des fins alimentaires s'observent quand les populations humaines locales ont besoin de protéines animales dans les régions où l'élevage est peu développé ou pauvre. Des espèces menacées par la déforestation et autres activités anthropiques deviennent sensibles aux prélèvements anarchiques. D'une manière générale, peu d'oiseaux acceptent les dérangements par l'homme lorsqu'ils s'installent pour nicher. Les dérangements que l'homme provoque dans l'exercice de ses activités vis-à-vis des oiseaux coloniaux conduisent aux déplacements voire même la disparation des espèces (Gill et al., 2001).

Le piégeage et l'abattage illicites des oiseaux se produisent partout en Afrique. C'est un problème reconnu en Algérie, en Libye, au Maroc et en Tunisie, mais les taux d'abattage les plus élevés sont enregistrés en Égypte. On estime qu'en moyenne 0,3 à 10,6 millions d'oiseaux sont tués illégalement en Égypte chaque année, faisant du pays l'endroit le plus dangereux pour les oiseaux migrateurs dans la Méditerranée (BirdLife International, 2018). Les oiseaux sont couramment piégés et abattus illégalement en R.D. Congo en général et à l'Est du pays en particulier.

Dans le massif d'Itombwe, globalement reconnu comme zone importante pour la conservation de la biodiversité (Plumptre et al., 2007), les perceptions des populations riveraines, les techniques de chasse, et les taxa couramment affectés n'ont jamais fait l'objet d'une quelconque recherche malgré les efforts de conservation prônés par le gouvernement central (MECNT, 2014).

Des tractations sont en cours entre des ONGs locales, le gouvernement et les 
communautés locales pour adopter un monde de conservation non conflictuel de la région pour une gestion durable des ressources naturelles d'une bonne partie du Massif d'Itombwe (Kujirakwinja et al., 2019) et la forêt de Burhinyi. Les communautés locales, ayant le plein droit de résider dans une zone précise et d'utiliser les ressources naturelles qui s'y trouvent, ne devraient pas être ignorées.

Des études menées en Afrique de l'Ouest (Sandjong Sani et al., 2013; Ilboudo et al., 2020) ont démontré la nécessité de tenir compte des perceptions locales dans la mise en œuvre des techniques de restauration et d'aménagement des écosystèmes.

Par ailleurs, il est largement reconnu que l'établissement d'une aire protégée doit se faire en collaboration étroite avec les communautés locales, en se rassurant que les droits de l'homme et les besoins socioéconomiques sont respectés durant tout le processus. Pour ce faire, les données de base fiables sur les impacts de l'établissement des aires protégées sont indispensables (West et al., 2006).

Cette étude évalue l'impact des principales activités humaines, des techniques de piégeage sur l'avifaune et les perçoivent de la population riveraine de la forêt de Burhinyi sur la conservation des oiseaux. Ceci permettrait aux parties prenantes de mieux planifier l'aménagement et la conservation de la forêt de Burhinyi en vue d'un développement local durable.

\section{MATERIEL ET METHODES \\ Zone d'étude}

La Chefferie de Burhinyi est située dans le Territoire de Mwenga, Province du SudKivu, dans la partie orientale de la République Démocratique du Congo (Figure 1). Elle est située à $83 \mathrm{~km}$ de Bukavu entre $2^{\circ} 45^{\prime}$ de latitude Sud et $28^{\circ} 33^{\prime}$ de longitude Est et sur une étendue de $328 \mathrm{~km}^{2}$ de surface. Elle présente un relief rigoureux de montagnes prolongeant la chaîne de Mitumba, et morphologiquement on distingue deux grandes sous-entités, qui sont : le Bas-Burhinyi et le Haut-Burhinyi comme grands ensembles sur le plan physique. Le Bas-Burhinyi, situé en dessous de $1800 \mathrm{~m}$ d'altitude, est entaillé des collines et des plateaux plus ou moins érodés. Le Haut-Burhinyi est au-delà de $1800 \mathrm{~m}$ d'altitude et abrite des montagnes imposantes dont les plus culminants sont : le mont Muhi : 3480 m, la chaîne de Chamirungu : $2568 \mathrm{~m}$ et la chaine de Ludge : $2415 \mathrm{~m}$. Burhinyi est subdivisé en 18 groupements comptant dans l'ensemble 54 localités. Ces groupements sont : Birhala, Bugobe, Buhogo, Chibindye, Chizuka, Ciriri, Cheshero, Itudu, Kalambo, Kitwabaluzi, Luhuku, Mulanga, Nirindja, Ntondo, Karhendezi, Cirere, Mulambi et Budaha (Ntamwira et Nyakabwa, 2008).

Le climat de cette entité, située entre 900 et $3480 \mathrm{~m}$ d'altitude, est équatorial. Ce climat se maintient régulier à cause de l'aspect physique (relief d'altitude) du milieu, des températures diverses et des directions des pluies.

L'étagement de la végétation dépend constamment du relief tel que signifié ci-haut et se répartit de la manière suivante : à $900 \mathrm{~m}$ d'altitude, une forêt équatoriale ; au-delà de $900 \mathrm{~m}$ se développe une forêt mésophile défrichée par l'homme pour des activités agropastorales et elle reste à l'état de vestige entre 1800 et $2400 \mathrm{~m}$ d'altitude. La forêt des bambous qui forme un couvert végétal sur les flancs et les sommets à plus de $2400 \mathrm{~m}$ d'altitude où elle subit une forte pression humaine à cause des cultures de maïs et de la pomme de terre ainsi que les coupes des tiges de Bambou (Sinarundinaria alpina) pour leur usage comme matériaux de construction. Actuellement, seuls les groupements de Cirere, Kalambo, Nirinja et de Chizuka possèdent encore des forêts ombrophiles d'altitude et une bambousaie au-dessus de $2000 \mathrm{~m}$ d'altitude sur les flancs des montagnes (Nangalire et al., 2017).

\section{Méthodes}

La méthode par questionnaire d'enquête a été utilisée pour collecter les données auprès de la population riveraine de la forêt de Burhinyi. Ainsi, un questionnaire d'enquête a été soumis à quelques habitants des villages autour de la forêt (Figure 2) afin d'évaluer les techniques de piégeage, la catégorie de la 
population impliquée et les taxons couramment capturés mais aussi recueillir leurs avis face à la problématique de la conservation des oiseaux et son habitat.

Le choix des enquêtés a été basé sur un seul critère à savoir :

- Être chef de ménage ou membre de ménage âgé de plus de dix-huit ans
Au total, 188 personnes ( 145 hommes et 43 femmes) issues de différents ménages ont été enquêtées dans 6 villages des groupements ciblés (Figure 2) dont 33 à Cirere, 32 à Cizuka, 32 à Ntondo, 31 à Luhuku, 30 à Nirinja et 30 à Kalambo.

L'analyse statistique des données et les tests de significativité sont faits avec les logiciels SPSS 20.0.

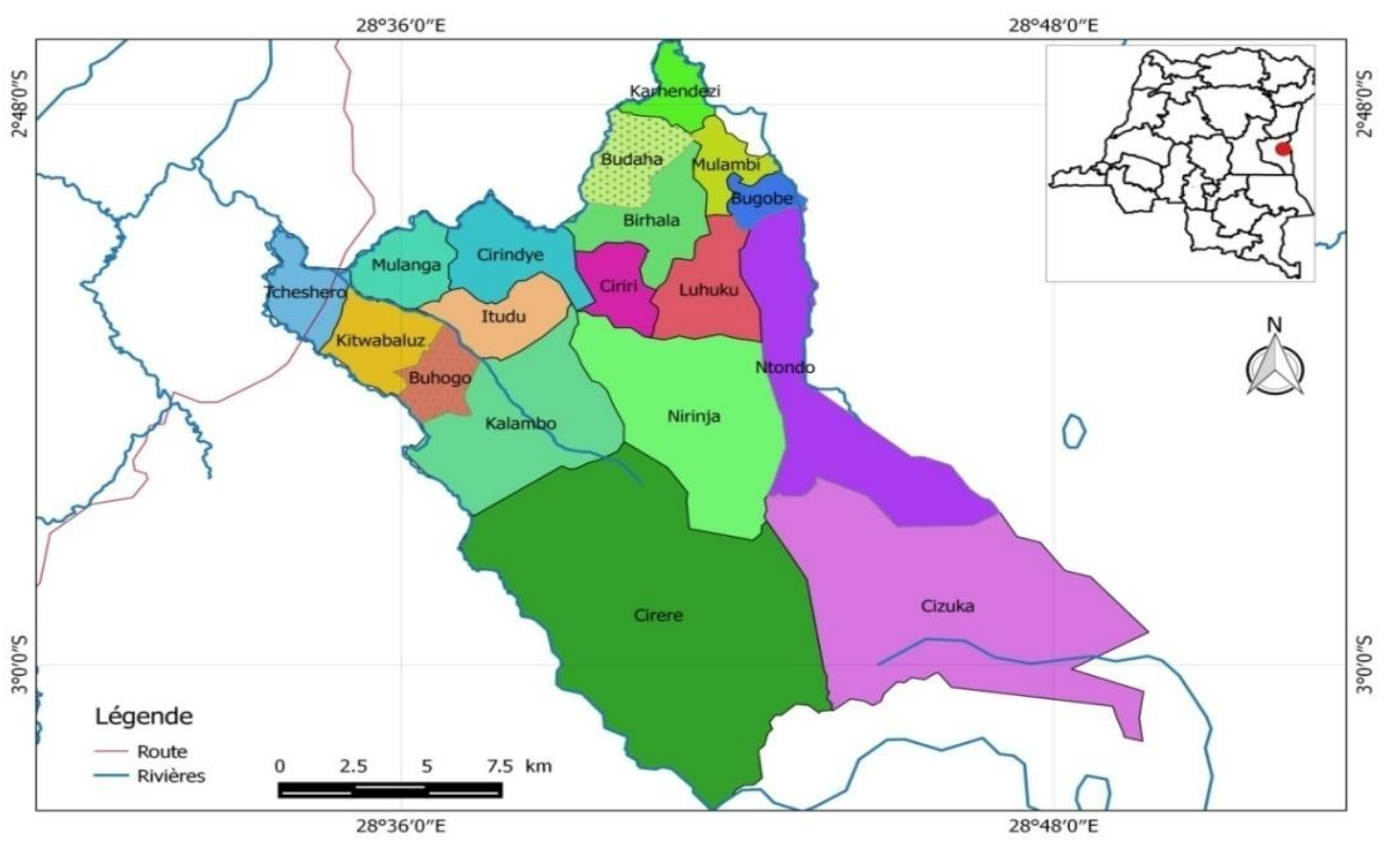

Figure 1 : Carte administrative de la chefferie de Burhinyi



Figure 2 : Localisation des villages d'échantillonnage. 


\section{RESULTATS}

\section{Profil identitaire des enquêtés}

Le Tableau 1 présente le profil identitaire des personnes enquêtées dans 6 groupements de la chefferie de Burhinyi. Ces enquêtés sont exclusivement adultes dont l'âge varie entre 18 et 78 ans. Le grand nombre des enquêtés se retrouve dans les classes d'âge comprises entre $20-29$ et $30-39$. Cependant, l'âge des enquêtés n'a pas significativement varié en fonction des groupements $(\mathrm{P}=0,504)$. Pour ce qui est de la répartition des enquêtés selon le genre, nous avons trouvé que les hommes étaient plus représentés parmi les habitats ciblés dans les 6 groupements. Il ressort que la majorité des enquêtés était des personnes mariées $(84,4 \%)$, suivie des célibataires qui ont représenté $11,2 \%$ des enquêtés. Il s'observe un taux d'analphabète le plus élevé dans ce milieu $(40,3 \%)$ avec un pic à Cirere $(78,8 \%)$. Les scolarisés du niveau primaire occupent la deuxième position avec $22,6 \%$ et en dernière position le seul universitaire (gradué) soit $0,11 \%$ des enquêtés. Notons que le niveau d'étude varie très significativement en fonction des groupements enquêtés $\left(\chi^{2}=44,69\right.$ avec $\left.P=0,001\right)$.

La taille de ménage a varié en fonction des groupements $(\mathrm{P}=0,035)$. Elle a présenté une moyenne de 7,1 à Nirinja, 6,6 à Cirere et Luhuku, 5,7 à Cizuka et enfin 5,2 à Kalambo.

\section{Activités anthropiques}

\section{Activités, professions principales et secondaires des enquêtés}

Les résultats du Tableau 2 présentent les principales activités humaines des enquêtés en fonction des groupements investigués. Il découle de ce Tableau 2 qu'au total, 7 différentes activités et deux professions sont exercées dans les six entités investiguées et dépendent très significativement $(\mathrm{P}=0,001)$ d'un groupement à un autre. L'agriculture occupe la première position avec un pic dans les activités humaines à Cirere. Elle est suivie successivement de l'enseignement, de l'exploitation minière, du petit commerce et de l'élevage. Il s'observe cependant, que Cirere avec un taux d'analphabète élevé, n'a pas d'enseignants mais la chasse, y reste l'activité secondaire importante. L'élevage, le petit commerce et la chasse sont des activités secondaires remarquables dans tous les groupements enquêtés. Par ailleurs, la variation des activités secondaires est hautement significative en fonctions des groupements.

\section{Abatage des oiseaux pour la consommation}

La Figure 3 montre que dans tous les groupements prospectés, les oiseaux sont couramment abattus pour la consommation. Le groupement de Cirere est toujours en tête dans la consommation des oiseaux. Il est suivi successivement par Nirinja, Cizuka et Ntondo à la dernière position. Par ailleurs, le test de Khi-deux montre des différences hautement significatives $(P=004)$ entre les groupements.

\section{Pression des activités anthropiques sur l'avifaune}

Techniques de piégeage des oiseaux dans les groupements riverains de la forêt de Burhinyi

Les résultats obtenus (Figure 4) sur les moyens de chasse des oiseaux dans notre milieu d'étude révèlent quatre principales techniques de capture (la colle artisanale, les filets japonais, la catapulte et les pièges artisanaux). Il s'observe que la technique de capture par les pièges artisanaux, la catapulte et la capture par les filets sont plus utilisées par nos enquêtés. Dans les groupements de Cirere, Kalambo et Nirinja, les pièges artisanaux sont plus utilisés, suivie des filets japonais tandis qu'à Cizuka, Luhuku et Ntondo c'est les catapultes qui viennent en première position. Cependant, les techniques de chasse ne sont pas significativement liées aux groupements $(\mathrm{P}=0,306)$.

\section{Couche de la population impliquée dans la chasse d'oiseaux}

Le Tableau 3 présente les couches de la population impliquées dans l'abatage d'oiseaux dans la zone d'étude. La lecture de ce Tableau 3 montre que deux catégories de la population sont impliquées dans l'abatage d'oiseaux dans notre zone d'étude. Il s'agit des hommes adultes et des jeunes garçons. Cette catégorie varie significativement selon les groupements $(p=0,0002)$. Nous constatons que seuls les hommes adultes chassent les oiseaux à Cirere et Nirinja tandis qu'à Ntondo 
et Luhuku c'est une activité presqu'exclusive aux jeunes garçons de moins de 18 ans.

\section{Catégorie d'oiseaux couramment capturée}

La Figure 5 présente les trois catégories d'oiseaux couramment capturés par les habitats de six groupements enquêtés. Le constat qui se dégage est que tous les oiseaux sans exception aucune sont habituellement capturés pour l'ensemble de la zone enquêtée. Cependant les préférences de capture varient très significativement $(\mathrm{P}=0,000)$ d'un groupement à l'autre. C'est ainsi qu'à Nirinja une chasse sélective est observée car les petits oiseaux n'y sont pas souvent piégés, tandis qu'à Ntondo ce sont les petits oiseaux qui sont plus concernés. Par ailleurs à Ntondo plusieurs enquêtés ne savent pas catégoriser les oiseaux chassés.

\section{Connaissances locales des taxons couramment chassés}

Les résultats dans le Tableau 4 révèlent que les populations locales enquêtées connaissent 22 taxa d'oiseaux couramment capturés dans la zone d'étude. Il s'observe toutefois une différence hautement significative $(P=0,000)$ dans ces connaissances selon les groupements. Parmi ces taxa, les Souimanga (Nectarinidae) sont les plus énumérés par les enquêtés. Ils sont suivis des Bulbuls (Pycnonotidae) et les Buses montagnardes (Accipitridae). Quarante-sept personnes soit $25 \%$ de nos enquêtés ne connaissent pas avec précision les taxons couramment abattus dans cette zone. Un test de Khi-deux a été appliqué pour vérifier si cette ignorance des taxa par une partie importante des enquêtés était liée au niveau d'étude, mais les résultats ont montré qu'il n'y avait pas de lien $\left(\chi^{2}=84,16\right.$ avec $\left.\mathrm{P}=0,801\right)$.

\section{Nombre d'oiseaux abattus journalièrement}

Les enquêtés ont estimé le nombre d'oiseaux tués par jour dans leurs villages. En moyenne 4,6 oiseaux sont abattus par jour dans six groupements enquêtés. Cependant le test non paramétrique de Kruskall-Wallis (Figure 6) montre des différences hautement significatives entre les six entités prospectées $(\mathrm{P}=0,000)$. Les oiseaux sont plus abattus dans le groupement de Cirere (moyenne d'oiseaux par jour : 7,3 $\pm 3,45)$, suivi de Nirinja
$(5,7 \pm 2,66)$, de Kalambo $(3,68 \pm 2,46)$ et enfin Ntondo $(2,28 \pm 2,2)$.

Nombre de chasseurs d'oiseaux connu par entité enquêtée

Le Tableau 5 présente le nombre de chasseurs d'oiseaux par entité investiguée.

Au total, 141 chasseurs d'oiseaux ont été identifiés dans les six entités enquêtées. Le nombre de chasseurs varie significativement selon le groupement et le grand nombre a été identifié dans Cirere (moyenne 5,64 $\pm 2,89$ ).

\section{Approches stratégiques pour la conservation de l'avifaune \\ Connaissances locales sur l'importance des oiseaux dans leurs milieux hormis la consommation}

L'évaluation des connaissances locales des enquêtés sur l'importance des oiseaux dans l'environnement est présentée dans le Tableau 6. Sur un total de 188 personnes enquêtées 106 soit $56,9 \%$ de nos enquêtés ne connaissent pas les rôles des oiseaux dans l'environnement. Environ $43,1 \%$ des enquêtés ont énuméré 9 rôles des oiseaux mis à part la consommation dans leurs milieux. Cependant, ces connaissances locales varient significativement d'une entité à l'autre $(\mathrm{p}=0,046)$. Le test de Khi-deux montre que les connaissances locales ne sont pas significativement liées au niveau d'études ( $\mathrm{p}=$ 0,06 ).

L'importance de la conservation des oiseaux a été exprimée par $80,1 \%$ des enquêtés (Tableau 7) alors que $14,5 \%$ ont dit qu'il n'était pas important de conserver l'avifaune et seulement $0,5 \%$ sans avis. Cette perception des enquêtés varie significativement en fonction des entités investiguées $(\mathrm{P}=0,000)$.

Connaissances ethno-ornithologiques des habitats enquêtés

Réponses des enquêtés sur l'usage culturel, symbolique et médicinal dans les groupements

Le Tableau 8 présente les nombres d'enquêtés suivant les catégories de réponses dans les différents groupements investigués. L'analyse montre que seulement 40 personnes soit $21,5 \%$ de la population enquêtée ont 
accepté que certaines espèces oiseaux sont utilisées comme symbole, dans la culture et dans la médecine traditionnelle à Burhinyi. Le test de Khi-deux révèle que les réponses de nos enquêtés dépendent de groupements $\left(\chi^{2}=20,62\right.$ avec $\mathrm{P}=0,023$ ).

\section{Reconnaissance locale des taxa d'oiseaux les plus utilisés}

Le Tableau 8 présente les désignations de chacun de taxa par les enquêtés selon les entités de prospection. Ces six taxons concernés ont été identifiés par $21 \%$ de la population enquêtée dans la zone d'étude. Les résultats du test de $\chi^{2}$ montrent que ces connaissances locales varient significativement selon les groupements.

\section{Principaux usages d'oiseaux horsmis la consommation}

Le tableau 9 présente les résultats de $20 \%$ des enquêtés qui connaissent les usages d'oiseaux à part la consommation dans la zone d'étude. Six principaux usages d'oiseaux sont révélés par les populations enquêtées. Dans cette zone quatre maladies (cardiaque, épilepsie, cicatrisation en cas de brûlures et stérilité) sont soignées par les organes des oiseaux. Les plumes et les griffes sont utilisées pour embellir les chapeaux des chefs des villages ou des groupements. Les résultats du test de $\chi^{2}$ montrent que les différents usages dépendent des groupements d'étude $\left(\chi^{2}=47,79\right.$ et $\mathrm{P}=0,021$ )

\section{Organes d'oiseaux utilisés dans la pharmacopée traditionnelle \\ Les enquêtés ont inventorié quatre organes d'oiseaux utilisés pendant les cérémonies et pour se soigner. Certains organes}

peuvent être combinés pour diverses utilisations. C'est le cas notamment des plumes et des griffes qui sont grillées et rendues en poudre pour cicatriser les brûlures. Les résultats dans le Tableau 10 montrent que les plumes et les griffes sont plus utilisés dans cette région. Cependant, l'utilisation d'organes varie en fonction des groupements $\left(\chi^{2}=50,84\right.$ avec un $\mathrm{P}=0,0406$ ).

\section{Perception des enquêtés sur le stock d'oiseaux dans la forêt}

Les avis des enquêtés sur le stock d'oiseaux dans la forêt sont partagés (Figure 7). Les enquêtés de deux groupements (Luhuku et Nirinja) pensent que la pression de la chasse diminue sensiblement les oiseaux dans la forêt tandis qu'à Cirere, Cizuka, Kalambo et Ntondo les habitats disent que l'avifaune augmente et qu'elle ne peut jamais s'épuiser.

\section{Stratégies formulées par les enquêtés pour la conservation de l'avifaune et son habitat}

Les habitats enquêtés ont formulé quelques mesures stratégiques pour la conservation des oiseaux et l'ensemble de la biodiversité de la forêt de Burhinyi (Tableau 11). Quoique onze mesures aient été proposées, quatre (l'abandon de la chasse, arrêt de la déforestation, sensibilisation de la population et la transformation de la forêt en parc) viennent de la majorité des enquêtés. Il s'observe que près de $16 \%$ des enquêtés n'ont pas formulé des mesures de conservation. Les résultats du test de $\chi^{2}$ révèlent que les réponses des enquêtés ne dépendent pas de leurs entités d'origine $\left(\chi^{2}=71,36\right.$ et $\left.\mathrm{P}=0,068\right)$.

Tableau 1 : Profil identitaire des enquêtés.

Groupements

\begin{tabular}{|c|c|c|c|c|c|c|c|c|c|c|}
\hline Paramètre & Modalité & Cirere & Cizuka & Kalambo & Luhuku & Nirinja & Ntondo & Total & $\chi^{2}$ & P-Value \\
\hline \multirow[t]{3}{*}{ Sexe } & Féminin & $27,3 \%$ & $25,0 \%$ & $14,3 \%$ & $25,8 \%$ & $26,7 \%$ & $12,5 \%$ & $22,0 \%$ & 3,99 & 0,55 \\
\hline & Masculin & $72,7 \%$ & $75,0 \%$ & $85,7 \%$ & $74,2 \%$ & $73,3 \%$ & $87,5 \%$ & $78,0 \%$ & & \\
\hline & Total & $100,0 \%$ & $100,0 \%$ & $100,0 \%$ & $100,0 \%$ & $100,0 \%$ & $100,0 \%$ & $100 \%$ & & \\
\hline \multirow{2}{*}{$\begin{array}{l}\text { Classe } \\
\text { d'âge }\end{array}$} & $<20$ & $0,0 \%$ & $6,3 \%$ & $3,6 \%$ & $0,0 \%$ & $6,7 \%$ & $0,0 \%$ & $2,7 \%$ & 29,3 & 0,504 \\
\hline & $20-29$ & $33,3 \%$ & $21,9 \%$ & $39,3 \%$ & $9,7 \%$ & $26,7 \%$ & $25,0 \%$ & $25,8 \%$ & & \\
\hline
\end{tabular}


B. C. MURHABALE et al. / Int. J. Biol. Chem. Sci. 14(6): 1999-2017, 2020

\begin{tabular}{|c|c|c|c|c|c|c|c|c|c|c|}
\hline & $30-39$ & $27,3 \%$ & $31,3 \%$ & $17,9 \%$ & $29,0 \%$ & $16,7 \%$ & $31,3 \%$ & $25,8 \%$ & & \\
\hline & $40-49$ & $18,2 \%$ & $15,6 \%$ & $7,1 \%$ & $29,0 \%$ & $13,3 \%$ & $25,0 \%$ & $18,3 \%$ & & \\
\hline & $50-59$ & $9,1 \%$ & $18,8 \%$ & $17,9 \%$ & $22,6 \%$ & $20,0 \%$ & $3,1 \%$ & $15,1 \%$ & & \\
\hline & $60+$ & $12,1 \%$ & $6,3 \%$ & $14,3 \%$ & $9,7 \%$ & $16,7 \%$ & $15,6 \%$ & $12,4 \%$ & & \\
\hline & Total & $100,0 \%$ & $100,0 \%$ & $100,0 \%$ & $100,0 \%$ & $100,0 \%$ & $100,0 \%$ & $100 \%$ & & \\
\hline \multirow[t]{5}{*}{ Etat civil } & Célibataire & $9,1 \%$ & $6,3 \%$ & $10,7 \%$ & $16,1 \%$ & $13,3 \%$ & $12,5 \%$ & $11,3 \%$ & 8,76 & 0,889 \\
\hline & Marié & $84,8 \%$ & $93,8 \%$ & $85,7 \%$ & $80,6 \%$ & $76,7 \%$ & $84,4 \%$ & $84,4 \%$ & & \\
\hline & Veuf & $3,0 \%$ & $0,0 \%$ & $0,0 \%$ & $0,0 \%$ & $3,3 \%$ & $3,1 \%$ & $1,6 \%$ & & \\
\hline & Veuve & $3,0 \%$ & $0,0 \%$ & $3,6 \%$ & $3,2 \%$ & $6,7 \%$ & $0,0 \%$ & $2,7 \%$ & & \\
\hline & Total & $100,0 \%$ & $100,0 \%$ & $100,0 \%$ & $100,0 \%$ & $100,0 \%$ & $100,0 \%$ & $100 \%$ & & \\
\hline \multirow{6}{*}{$\begin{array}{l}\text { Niveau } \\
\text { d'étude }\end{array}$} & Diplômé d'état & $0,0 \%$ & $21,9 \%$ & $17,9 \%$ & $25,8 \%$ & $3,3 \%$ & $25,0 \%$ & $15,6 \%$ & 44,7 & 0,001 \\
\hline & Gradué & $0,0 \%$ & $0,0 \%$ & $0,0 \%$ & $0,0 \%$ & $0,0 \%$ & $3,1 \%$ &, $5 \%$ & & \\
\hline & Secondaires & $9,1 \%$ & $15,6 \%$ & $28,6 \%$ & $29,0 \%$ & $20,0 \%$ & $25,0 \%$ & $21,0 \%$ & & \\
\hline & Primaire & $12,1 \%$ & $18,8 \%$ & $21,4 \%$ & $25,8 \%$ & $33,3 \%$ & $25,0 \%$ & $22,6 \%$ & & \\
\hline & Analphabete & $78,8 \%$ & $43,8 \%$ & $32,1 \%$ & $19,4 \%$ & $43,3 \%$ & $21,9 \%$ & $40,3 \%$ & & \\
\hline & Total & $100,0 \%$ & $100,0 \%$ & $100,0 \%$ & $100,0 \%$ & $100,0 \%$ & $100,0 \%$ & $100 \%$ & & \\
\hline
\end{tabular}

Tableaux 2 : Répartition des activités principales et secondaires des enquêtés dans les Groupements.

\begin{tabular}{|c|c|c|c|c|c|c|c|c|c|}
\hline $\begin{array}{l}\text { Activités } \\
\text { principales }\end{array}$ & Cirere & Cizuka & Kalambo & Luhuku & Nirinja & Ntondo & Total & $\chi^{2}$ & P-Value \\
\hline Agriculture & $90,9 \%$ & $56,3 \%$ & $67,9 \%$ & $45,2 \%$ & $76,7 \%$ & $25,0 \%$ & $60,2 \%$ & \multirow[t]{8}{*}{58,56} & \multirow{8}{*}{0,001} \\
\hline Elevage & $3,0 \%$ & $0,0 \%$ & $0,0 \%$ & $9,7 \%$ & $3,3 \%$ & $9,4 \%$ & $4,3 \%$ & & \\
\hline Enseignement & $0,0 \%$ & $21,9 \%$ & $14,3 \%$ & $22,6 \%$ & $3,3 \%$ & $21,9 \%$ & $14,0 \%$ & & \\
\hline $\begin{array}{l}\text { Exploitation } \\
\text { minière }\end{array}$ & $3,0 \%$ & $12,5 \%$ & $14,3 \%$ & $3,2 \%$ & $10,0 \%$ & $28,1 \%$ & $11,8 \%$ & & \\
\hline $\begin{array}{l}\text { Fabrication des } \\
\text { assiettes } \\
\text { traditionnelles }\end{array}$ & $0,0 \%$ & $3,1 \%$ & $0,0 \%$ & $0,0 \%$ & $0,0 \%$ & $0,0 \%$ &, $5 \%$ & & \\
\hline Pasteur d'église & $0,0 \%$ & $3,1 \%$ & $0,0 \%$ & $3,2 \%$ & $0,0 \%$ & $3,1 \%$ & $1,6 \%$ & & \\
\hline $\begin{array}{l}\text { Petit } \\
\text { commerce }\end{array}$ & $3,0 \%$ & $3,1 \%$ & $3,6 \%$ & $16,1 \%$ & $6,7 \%$ & $12,5 \%$ & $7,5 \%$ & & \\
\hline Total & $100,0 \%$ & $100,0 \%$ & $100,0 \%$ & $100,0 \%$ & $100,0 \%$ & $100,0 \%$ & $100,0 \%$ & & \\
\hline \multicolumn{10}{|c|}{ Activités secondaires } \\
\hline Agriculture & $21,2 \%$ & $37,5 \%$ & $7,1 \%$ & $32,3 \%$ & $23,3 \%$ & $40,6 \%$ & $27,4 \%$ & \multirow[t]{8}{*}{107,9} & \multirow[t]{8}{*}{0,0004} \\
\hline Chasse & $18,2 \%$ & $0,0 \%$ & $0,0 \%$ & $0,0 \%$ & $0,0 \%$ & $0,0 \%$ & $3,2 \%$ & & \\
\hline Elevage & $33,3 \%$ & $34,4 \%$ & $57,1 \%$ & $12,9 \%$ & $23,3 \%$ & $9,4 \%$ & $28,0 \%$ & & \\
\hline Enseignement & $0,0 \%$ & $0,0 \%$ & $7,1 \%$ & $0,0 \%$ & $0,0 \%$ & $0,0 \%$ & $1,1 \%$ & & \\
\hline $\begin{array}{l}\text { Exploitation } \\
\text { artisanale du } \\
\text { bois }\end{array}$ & $6,1 \%$ & $0,0 \%$ & $3,6 \%$ & $0,0 \%$ & $3,3 \%$ & $0,0 \%$ & $2,2 \%$ & & \\
\hline $\begin{array}{l}\text { Exploitation } \\
\text { minière }\end{array}$ & $12,1 \%$ & $0,0 \%$ & $3,6 \%$ & $3,2 \%$ & $16,7 \%$ & $3,1 \%$ & $6,5 \%$ & & \\
\hline Pasteur d'église & $0,0 \%$ & $0,0 \%$ & $0,0 \%$ & $3,2 \%$ & $3,3 \%$ & $3,1 \%$ & $1,6 \%$ & & \\
\hline & & & & 2006 & & & & & \\
\hline
\end{tabular}


B. C. MURHABALE et al. / Int. J. Biol. Chem. Sci. 14(6): 1999-2017, 2020

Petit

$6,1 \% \quad 21,9 \% \quad 21,4 \% \quad 48,4 \% \quad 23,3 \% \quad 31,3 \% \quad 25,3 \%$

commerce

Aucune

$3,0 \% \quad 6,3 \%$

$0,0 \%$

$0,0 \%$

$6,7 \%$

$12,4 \% \quad 4,7 \%$

Total

$100,0 \% \quad 100,0 \%$

$100,0 \%$

$100,0 \% \quad 100,0 \% \quad 100,0 \% \quad 100,0 \%$

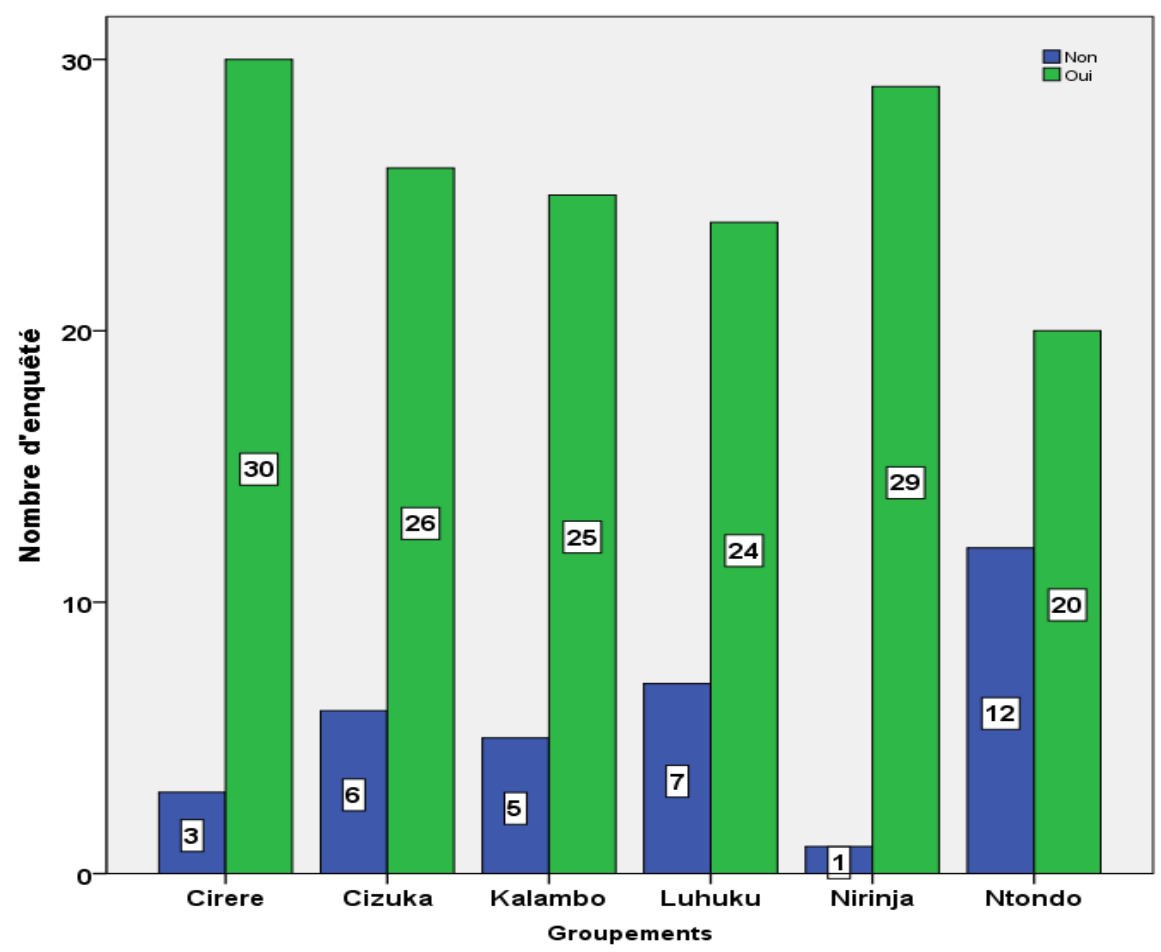

Figure 3 : Etat de la consommation des oiseaux dans les entités enquêtées.



Figure 4 : Technique d'abatage d'oiseaux dans les différents groupements d'enquête. 
Tableau 3 : Repartions des chasseurs d'oiseaux par groupements investigués.

\begin{tabular}{lccccc}
\hline Groupements & Hommes & Jeunes garçons & Ne sais pas & $\chi^{2}$ & P-Value \\
Cirere & $90,9 \%$ & $0 \%$ & $9,1 \%$ & 107,004 & 0,0002 \\
Cizuka & $21,9 \%$ & $59,4 \%$ & $18,8 \%$ & & \\
Kalambo & $73,3 \%$ & $13,3 \%$ & $13,3 \%$ & & \\
Luhuku & $12,9 \%$ & $64,5 \%$ & $22,6 \%$ & & \\
Nirinja & $96,7 \%$ & & $3,3 \%$ & & \\
Ntondo & $9,4 \%$ & $56,3 \%$ & $34,4 \%$ & & \\
\hline
\end{tabular}



Figure 5 : Catégories d'oiseaux couramment capturées dans la zone d'étude.

Tableau 4 : Répartition par groupement des connaissances locales sur les taxa couramment capturés à Burhinyi.

\begin{tabular}{lcccccccc}
\hline Taxons & Cirere & Cizuka & Kalambo & Luhuku & Nirinja & Ntondo & $\boldsymbol{\chi}^{2}$ & P-Value \\
\hline Aigle & 0 & 0 & 0 & 0 & 5 & 0 & 232,6 & 0,000 \\
Amarante & 2 & 0 & 1 & 1 & 3 & 0 & & \\
Bulbul & 4 & 5 & 3 & 7 & 4 & 3 & \\
Buse d'Afrique & 0 & 0 & 3 & 0 & 1 & 0 & \\
Buse montagnardes & 5 & 1 & 4 & 0 & 6 & 0 & \\
Coliou rayé & 4 & 2 & 1 & 0 & 0 & 2 & \\
Eperviers & 0 & 0 & 0 & 1 & 4 & 1 & \\
Estrild & 1 & 0 & 0 & 0 & 0 & 0 & \\
Francolin & 2 & 0 & 1 & 0 & 0 & 0 & \\
Gonolek de montagne & 1 & 0 & 0 & 0 & 0 & 0 & \\
Grive & 0 & 0 & 1 & 0 & 0 & 0 & & \\
Hirondelles & 0 & 0 & 0 & 0 & 2 & 0 &
\end{tabular}




$\begin{array}{lcccccc}\text { Merles } & 0 & 1 & 0 & 0 & 0 & 0 \\ \text { Moineaux } & 0 & 0 & 0 & 1 & 0 & 0 \\ \text { Pigeon rameron } & 4 & 0 & 0 & 0 & 0 & 0 \\ \text { Senegali } & 0 & 0 & 2 & 0 & 0 & 0 \\ \text { Souimanga } & 3 & 7 & 6 & 7 & 3 & 6 \\ \text { Tisserins } & 0 & 2 & 1 & 1 & 0 & 1 \\ \text { Touraco de ruwenzori } & 4 & 0 & 1 & 0 & 0 & 0 \\ \text { Touraco géant } & 1 & 0 & 0 & 0 & 0 & 0 \\ \text { Tourterelle } & 0 & 1 & 0 & 1 & 1 & 0 \\ \text { Zosterops } & 0 & 3 & 0 & 0 & 0 & 0 \\ \text { Ne connais pas } & 2 & 10 & 3 & 12 & 1 & 19\end{array}$



Figure 6 : Nombre d'oiseaux abattus journalièrement dans six groupements de Burhinyi.

Tableau 5 : Nombre connu des chasseurs d'oiseaux par groupement.

\begin{tabular}{lcccc}
\hline Groupement & Nombre des Chasseurs & Moyenne \pm SD & F & P-Value \\
\hline Cirere & 28 & $5,64 \pm 2,89$ & 9,560 & 0,0007 \\
Cizuka & 21 & $3,38 \pm 1,35$ & & \\
Kalambo & 23 & $2,74 \pm 1,35$ & \\
Luhuku & 22 & $2,18 \pm 1,25$ & \\
Nirinja & 27 & $4,11 \pm 1,92$ & \\
Ntondo & 20 & $3,65 \pm 2,13$ & \\
\end{tabular}


Tableau 6 : Importance des oiseaux dans le milieu à part la consommation.

\begin{tabular}{|c|c|c|c|c|c|c|c|c|}
\hline Paramètres & Cirere & Cizuka & Kalambo & Luhuku & Nirinja & Ntondo & $\chi^{2}$ & P-Value \\
\hline Non & 17 & 19 & 15 & 26 & 8 & 21 & 22,73 & 0,011 \\
\hline Oui & 14 & 12 & 12 & 4 & 19 & 10 & & \\
\hline \multicolumn{9}{|c|}{ Importances des oiseaux selon les enquêtés } \\
\hline C'est une richesse du pays & 1 & 0 & 0 & 1 & 0 & 0 & 62,04 & 0,046 \\
\hline Dissémination des graines & 0 & 1 & 0 & 0 & 1 & 2 & & \\
\hline Ils attirent les chercheurs & 0 & 0 & 1 & 0 & 0 & 0 & & \\
\hline Ils chantent bien & 1 & 3 & 3 & 1 & 1 & 1 & & \\
\hline Ils nous réveillent le matin & 5 & 0 & 1 & 0 & 3 & 1 & & \\
\hline Indicateur de l'heure & 0 & 0 & 1 & 0 & 1 & 0 & & \\
\hline $\begin{array}{l}\text { Mangent les insectes } \\
\text { nuisibles }\end{array}$ & 6 & 8 & 6 & 2 & 11 & 6 & & \\
\hline Pollinisation & 1 & 0 & 0 & 0 & 0 & 0 & & \\
\hline $\begin{array}{l}\text { Utilisation comme } \\
\text { médicament }\end{array}$ & 0 & 0 & 0 & 0 & 2 & 0 & & \\
\hline \multicolumn{9}{|c|}{ Est-il important de conserver les oiseaux de votre forêt? } \\
\hline Non & 3 & 2 & 0 & 15 & 4 & 3 & 38,69 & 0,000 \\
\hline Oui & 28 & 28 & 27 & 16 & 24 & 26 & & \\
\hline
\end{tabular}

Tableau 7 : Aperçu sur l'usage culturel, Symbolique et médicinal des oiseaux dans la zone d'étude.

\begin{tabular}{lcccccccc}
\hline \multicolumn{1}{c}{ Modalité } & Cirere & Cizuka & Kalambo & Luhuku & Nirinja & Ntondo & $\chi^{\mathbf{2}}$ & P-Value \\
\hline Non & 18 & 26 & 22 & 27 & 16 & 27 & 20,62 & 0,023 \\
Oui & 13 & 4 & 4 & 4 & 11 & 4 & & \\
Ne sais pas & 2 & 2 & 4 & 0 & 3 & 1 & & \\
\hline
\end{tabular}

Tableau 8: Désignation des taxa d'oiseaux d'usage symbolique, culturel et médicinale dans la chefferie de Burhinyi.

\begin{tabular}{lcccccccc}
\hline Taxa & Cirere & Cizuka & Kalambo & Luhuku & Nirinja & Ntondo & $\chi^{\mathbf{2}}$ & P-Value \\
\hline Aigle & 6 & 0 & 2 & 2 & 2 & 1 & 47,98 & 0,019 \\
Buse montagnarde & 3 & 1 & 0 & 0 & 5 & 0 & & \\
Corbeau & 1 & 0 & 2 & 0 & 0 & 1 & \\
Gonolek de montagne & 0 & 1 & 0 & 0 & 0 & 0 & \\
Hibou & 0 & 2 & 0 & 1 & 1 & 0 & \\
Touraco de ruwenzori & 3 & 0 & 0 & 1 & 3 & 2 & \\
Ne sais pas & 20 & 28 & 26 & 27 & 19 & 28 & \\
& & & & & & & & \\
\hline
\end{tabular}


Tableau 9 : Usages d'oiseaux en dehors de la consommation dans la chefferie de Burhinyi.

\begin{tabular}{|c|c|c|c|c|c|c|c|c|c|}
\hline \multirow[t]{2}{*}{ Groupement } & \multicolumn{7}{|c|}{ Usages } & \multirow{2}{*}{$\frac{\chi^{2}}{47,98}$} & \multirow{2}{*}{$\frac{\text { P-Value }}{0,019}$} \\
\hline & $\begin{array}{c}\text { Cicatrisant } \\
\text { brûlure }\end{array}$ & Epilepsie & $\begin{array}{c}\text { Maladie } \\
\text { cardiaque }\end{array}$ & Stérilité & Cérémonie & $\begin{array}{l}\text { Chapeau } \\
\text { des chef }\end{array}$ & $\begin{array}{c}\text { Ne sais } \\
\text { pas }\end{array}$ & & \\
\hline Cirere & $3,00 \%$ & $0 \%$ & $9,10 \%$ & $0 \%$ & $6,10 \%$ & $18,20 \%$ & $63,60 \%$ & & \\
\hline Cizuka & $0 \%$ & $6,30 \%$ & $3,10 \%$ & $3,10 \%$ & $0 \%$ & $0 \%$ & $87,50 \%$ & & \\
\hline Kalambo & $6,70 \%$ & $0 \%$ & $0 \%$ & $0 \%$ & $0 \%$ & $6,70 \%$ & $86,70 \%$ & & \\
\hline Luhuku & $0 \%$ & $3,20 \%$ & $0 \%$ & $0 \%$ & $3,20 \%$ & $6,50 \%$ & $87,10 \%$ & & \\
\hline Nirinja & $0 \%$ & $3,30 \%$ & $16,70 \%$ & $0 \%$ & $10,00 \%$ & $3,30 \%$ & $66,70 \%$ & & \\
\hline Ntondo & $3,10 \%$ & $0 \%$ & $0 \%$ & $0 \%$ & $6,30 \%$ & $3,10 \%$ & $87,50 \%$ & & \\
\hline Total & $2,10 \%$ & $2,10 \%$ & $4,80 \%$ & $0,50 \%$ & $4,30 \%$ & $6,40 \%$ & $79,80 \%$ & & \\
\hline
\end{tabular}

Tableau 10 : Organes d'oiseaux utilisés pars les enquêtés

\begin{tabular}{lcccccccc}
\hline Organe utilisé & Cirere & Cizuka & Kalambo & Luhuku & Nirinja & Ntondo & $\chi^{\mathbf{2}}$ & P-Value \\
\hline Cœur & $0 \%$ & $50,0 \%$ & $0 \%$ & $25,0 \%$ & $25,0 \%$ & $0 \%$ & 50,84 & 0,0406 \\
Griffe & $33,3 \%$ & $11,1 \%$ & $0 \%$ & $0 \%$ & $55,6 \%$ & $0 \%$ & & \\
Plumes & $25,0 \%$ & $0 \%$ & $0 \%$ & $12,5 \%$ & $37,5 \%$ & $25,0 \%$ & & \\
Plumes et griffe & $43,8 \%$ & $0 \%$ & $25,0 \%$ & $12,5 \%$ & $6,3 \%$ & $12,5 \%$ & & \\
Tête & & $100,0 \%$ & & & & & & \\
Ne sais pas & $14,0 \%$ & $18,7 \%$ & $17,3 \%$ & $18,0 \%$ & $13,3 \%$ & $18,7 \%$ & & \\
& $17,6 \%$ & $17,0 \%$ & $16,0 \%$ & $16,5 \%$ & $16,0 \%$ & $17,0 \%$ & & \\
\hline
\end{tabular}

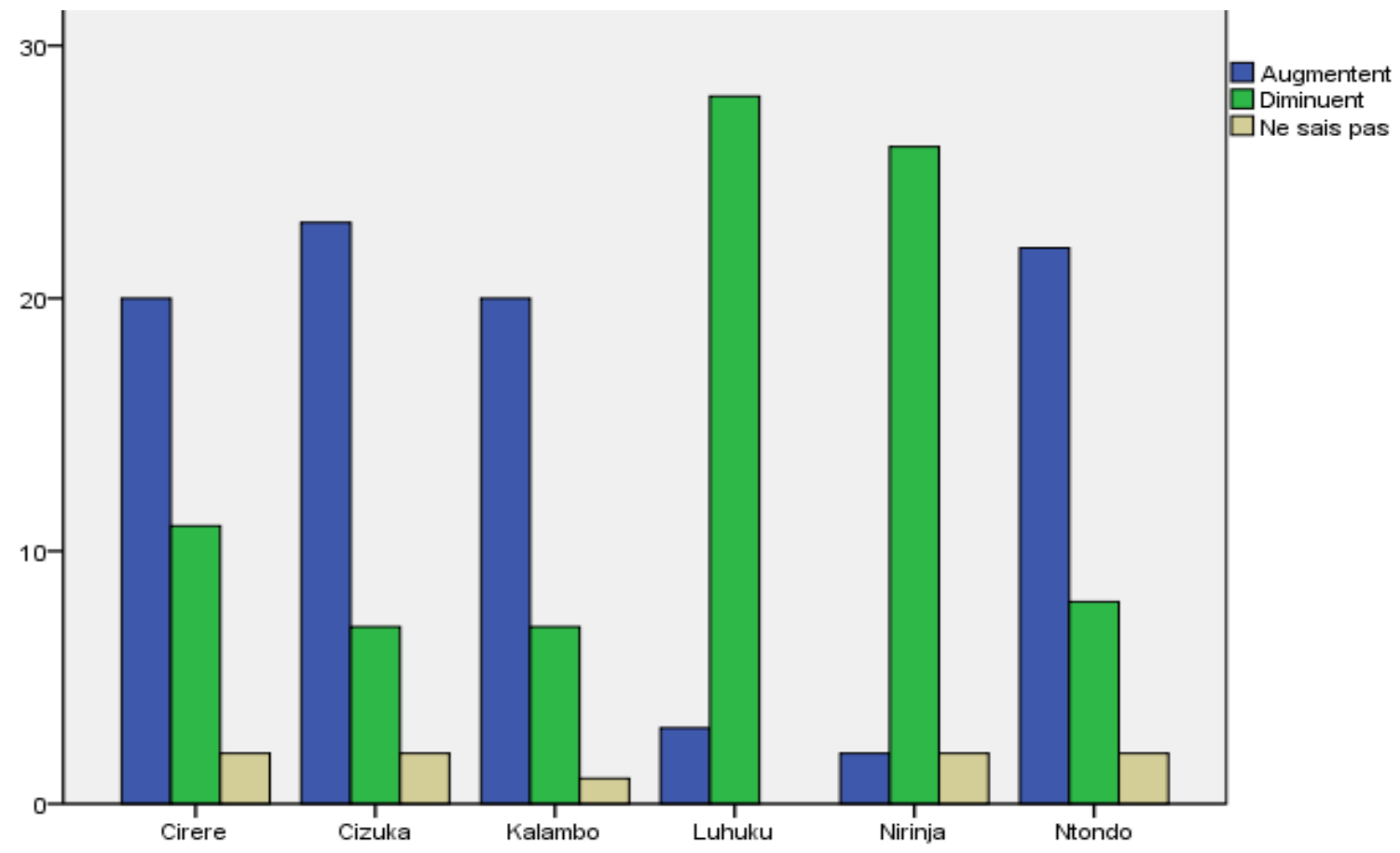

Figure 7 : Perception des enquêtés sur le stock d'oiseaux dans la forêt. 
Tableau 11 : Mesures stratégiques pour la conservation des oiseaux dans la forêt de Burhinyi.

\begin{tabular}{lcccccccc}
\hline $\begin{array}{l}\text { Mesures proposées par } \\
\text { les enquêtés }\end{array}$ & Cirere & Cizuka & Kalambo & Luhuku & Nirinja & Ntondo & $\chi^{2}$ & P-Value \\
\hline Abandonner la chasse & $7,7 \%$ & $53,8 \%$ & $7,7 \%$ & $15,4 \%$ & $0 \%$ & $15,4 \%$ & 77,69 & 0,0002 \\
$\begin{array}{l}\text { Donner l'emploi aux } \\
\text { chasseurs }\end{array}$ & $27,3 \%$ & $0 \%$ & $13,6 \%$ & $0 \%$ & $59,1 \%$ & & & \\
$\begin{array}{l}\text { Mettre fin à la } \\
\text { déforestation }\end{array}$ & $0 \%$ & $44,4 \%$ & $11,1 \%$ & $11,1 \%$ & $22,2 \%$ & $11,1 \%$ & \\
$\begin{array}{l}\text { Sensibiliser la } \\
\text { population }\end{array}$ & $15,6 \%$ & $21,9 \%$ & $15,6 \%$ & $12,5 \%$ & $6,3 \%$ & $28,1 \%$ & \\
$\begin{array}{l}\text { Transformer cette forêt } \\
\text { en Parc }\end{array}$ & $17,2 \%$ & $3,4 \%$ & $20,7 \%$ & $37,9 \%$ & $10,3 \%$ & $10,3 \%$ & \\
Aucune & $19,3 \%$ & $15,7 \%$ & $16,9 \%$ & $15,7 \%$ & $12,0 \%$ & $20,5 \%$ & & \\
\hline
\end{tabular}

\section{DISCUSSION}

\section{Activités anthropiques}

La recherche des facteurs anthropiques susceptibles d'agir sur le fonctionnement et le devenir des populations animales est un des enjeux mangeurs en biologie de la conservation. De nombreuses activités humaines peuvent provoquer du dérangement et affecter la faune sauvage (Tamisier et al., 2003 ; Gallai et al., 2009). Pour orienter et accompagner les populations locales de Burhinyi dans leurs soucis de la conservation de la biodiversité, il a fallu tout premièrement documenter les activités humaines à impact négatif sur l'avifaune. Nos résultats ont relevé 9 activités anthropiques qui dépendent hautement des groupements (Khi-deux $=58,56$ et $\mathrm{P}=0,001)$ de la région d'études ciblée (Tableau 2). L'agriculture est l'activité humaine principale la plus importante dans les six groupements investigués. Elle est plus pratiquée à Cirere où la population est majoritairement analphabète. Les enquêtés ont relevé que l'élevage, la chasse, l'exploitation minière et le petit commerce constituent leurs activités secondaires les plus remarquables. Parmi les 9 activités, trois (l'agriculture, la chasse, l'exploitation minière) sont connues pour l'impact négatif sur la biodiversité. D'après Barnosky et al. (2011) la perte de l'habitat à travers l'agriculture, la chasse et l'exploitation des ressources naturelles conduisent à l'appauvrissement spécifique et quantitatif de la faune. Deikumah et al. (2017) a démontré l'effet négatif de l'agriculture sur les espèces forestières en milieu tropical. L'agriculture itinérante sur brulis telle que pratiquée à Burhinyi conduit à la déforestation totale des collines. L'exploitation artisanale des minerais en forêt conduit à la fragmentation plus ou moins prononcée de l'habitat. Si rien n'est fait pour stopper ou réduire l'ampleur de ces activités à Burhinyi, le pire est à craindre pour la biodiversité et la valeur globale du site.

Les résultats sur la chasse des oiseaux ont montré que dans tous les groupements prospectés, les oiseaux sont couramment abattus pour la consommation. Le test de Khideux a montré que l'abatage des oiseaux dépend significativement des groupements. C'est ainsi que le groupement de Cirere est en tête dans la consommation des oiseaux. Il est suivi successivement par Nirinja et Cizuka. Parmi les principales menaces sur les espèces d'oiseaux dans le monde, les activités illicites telles que les piégeages et la chasse sont des pratiques courantes en Afrique ((BirdLife International, 2018).

\section{Impact de la chasse sur l'avifaune à Burhinyi}

Nos résultats ont déniché quatre techniques de chasse d'oiseaux (Catapulte, Pièges artisanaux, Filets japonais et la glue 
artisanale) dans la zone d'étude. Quoique les quatre techniques soient présentes dans les six groupements investigués, nous avons observé que les pièges artisanaux sont plus utilisés à Cirere, Nirinja et Kalambo. Ces pièges sont faits par les fils métalliques ou soit par des cordes faites à partir d'écorces des lianes. Les filets japonais sont faiblement utilisés à Luhuku et à Ntondo où les catapultes occupent la première place.

Sachant les effets négatifs que peuvent avoir ces deux techniques (pièges et filets) nondiscriminatoires sur l'avifaune, les activités de conservation de l'avifaune, notamment la sensibilisation devraient se concentrer dans le groupement de Cirere, Nirinja et Kalambo.

Les résultats présentés dans le Tableau 3 ont montré que la chasse d'oiseaux est une activité exclusive des hommes adultes et des jeunes garçons de moins de 18 ans. Aucune femme n'est citée par nos enquêtés dans ce genre d'activité. Dans certains groupements (Cirere, Kalambo et Nirinja) ce sont les hommes adultes qui sont plus impliqués tandis qu'à Luhuku, Ntondo et Cizuka ce sont les jeunes garçons qui dominent. Cette situation pourrait être expliquée par le fait que les trois premiers groupements sont très proches de la forêt mais aussi par les moyens ou les techniques de chasses utilisées dans les différentes entités. Par ailleurs, les études sur les viandes de brousse en Afrique ont montré que la chasse des gibiers est une activité principalement masculine au moment où les ventes des viandes des brousses sur les marchés sont quasi exclusivement féminines (Edderai et Dame, 2006 ; Mbete et al., 2011).

Il découle des résultats présentés par la Figure 5 que tous les oiseaux sans exception aucune sont habituellement abattus dans les groupements enquêtés. La catégorie d'oiseaux chassée varie très significativement $(\mathrm{P}=0,000)$ d'un groupement à l'autre. C'est ainsi qu'à Nirinja une chasse sélective est observée car les petits oiseaux n'y sont pas souvent piégés, tandis qu'à Ntondo ce sont plutôt les petits oiseaux qui sont plus concernés. La situation de Ntondo s'expliquerait par la grande intensité de déforestation qui s'y passe depuis quelques décennies, conduisant ainsi à l'absence actuelle de gros oiseaux et par conséquent la population se rabat aux petits oiseaux.

L'évaluation des connaissances locales sur les taxa d'oiseaux chassés ou piégés dans cette zone fait état de 22 taxons bien connus. Toutefois, une différence hautement significative $(\mathrm{P}=0,000)$ dans ces connaissances a été observée selon les groupements. Parmi les taxa connus par les enquêtés, les Souimanga (Nectarinidae) sont les plus énumérés. Ils sont suivis des Bulbuls (Pycnonotidae) et des Buses montagnardes (Accipitridae). Cependant, près de 47 personnes soit $25 \%$ des enquêtés ne savent pas avec exactitude les taxons couramment abattus dans cette zone. Les populations enquêtées peuvent identifier jusqu'au niveau de l'espèce les gros oiseaux (cas du Touraco de Ruwenzori) mais pour les petits oiseaux ils se limitent soit à la famille ou soit au niveau du genre.

Quant à la quantité d'oiseaux abattue journalièrement, les enquêtés ont estimé qu'en moyenne 4,6 oiseaux sont abattus par jour dans six groupements investigués. Le test non paramétrique de Kruskall-Wallis a montré des différences hautement significatives entre les six entités prospectées $(\mathrm{P}=0,000)$. Il a été observé que les oiseaux sont plus abattus dans le groupement de Cirere (moyenne d'oiseaux par jour : 7,3 $\pm 3,45)$, suivi de Nirinja $(5,7 \pm 2,66)$, de Kalambo $(3,68 \pm 2,46)$ et enfin Ntondo $(2,28 \pm 2,2)$. En moyenne, on estime que 0,3 à 10,6 millions d'oiseaux sont tués illégalement chaque année en Egypte. Ce pays est considéré comme étant le plus dangereux pour les oiseaux migrateurs dans la Méditerranée (Brochet et al., 2016). En Europe, l'abattage illicite annuel est estimé à environ 2,1 millions d'oiseaux (Brochet et al., 2019).

Au total, 141 chasseurs d'oiseaux ont été identifiés dans les six entités enquêtées. Le nombre de chasseurs varie significativement selon le groupement et le grand nombre a été identifié dans le groupement de Cirere (moyenne 5,64 $\pm 2,89$ ).

\section{Approches stratégiques pour la conservation de l'avifaune}

Les résultats présentés dans le Tableau

6 révèlent les connaissances locales des 
enquêtés sur l'importance des oiseaux dans l'environnement. Environs 106 soit 56,9\% de nos enquêtés ne connaissent pas les rôles des oiseaux dans l'environnement. Les enquêtés ont énuméré 9 rôles des oiseaux dans leurs milieu mis à part la consommation. Il s'observe que ces connaissances locales varient significativement en fonction des entités enquêtées $(p=0,046)$. Parmi le rôle des oiseaux dans l'environnement, un grand nombre des enquêtés ont affirmé que les oiseaux mangent les insectes nuisibles à l'homme et aux plantes. Ces observations de nos enquêtés rencontrent celles de Ndang'ang'a et al. (2013) qui stipulent que les oiseaux insectivores contribuent à la lutte contre l'infestation par les animaux nuisibles dans le champ. L'importance de la conservation des oiseaux a été exprimée par $80,1 \%$ des enquêtés (Tableau 7) alors que $14,5 \%$ ont dit qu'il n'était pas important de conserver l'avifaune et seulement $0,5 \%$ sans avis. Cette perception des enquêtés varie significativement en fonction des entités investiguées $(\mathrm{P}=0,000)$. La majorité des enquêtés ne voudraient pas la disparition de l'avifaune dans leur milieu à cause des multiples rôles socio-écologiques qu'on lui reconnait. Cette attitude de la population enquêtée prouve que la conservation de l'avifaune dans la chefferie de Burhinyi peut être facilement acceptée.

En rapport avec les connaissances ethnoornithologiques, 40 personnes soit 21,5\% de la population enquêtée ont accepté que certaines espèces oiseaux (ou leurs organes) sont utilisées comme symbole, dans la culture et dans la médecine traditionnelle à Burhinyi. Le test de Khi-deux révèle que les réponses de nos enquêtés dépendent de groupements $\left(\chi^{2}=20,62\right.$ et $\left.\mathrm{P}=0,023\right)$. Six taxa (Aigle, Hibou, Corbeau, Buses montagnardes, Gonolek de montagne et Touraco de Ruwenzori) cités par les enquêtés sont utilisés pour soigner quatre maladies mais aussi embellir les chapeaux des chefs de villages qu'ils portent pendant les cérémonies coutumières. Quatre organes d'oiseaux utilisés pendant les cérémonies et pour se soigner ont été inventoriés par les enquêtés. Dans ces entités investiguées, certains organes peuvent être combinés pour diverses utilisations. C'est le cas notamment des plumes et des griffes qui sont grillés et rendus en poudre pour cicatriser les brûlures. Au sud du Benin Lougbégno (2016) a trouvé que tous les organes de cinq espèces d'oiseaux gibiers (le dendrocygne veuf : Dendrocygna viduata, la grande aigrette: Egretta alba, l'aigrette ardoisée : Egretta ardesiaca, la talève d'Allen : Porphyrio alleni et la talève sultane : Porphyrio porphyrio) étaient utilisés en pharmacopée traditionnelle.

Néanmoins, nos résultats sur les connaissances ethnoornithologiques obtenus devraient être considérés avec prudence, car ils proviennent d'uniquement $20 \%$ de nos enquêtés. Des études plus approfondies sur les valeurs d'usages et les déterminants socioéconomiques liés à l'exploitation des oiseaux dans la chefferie de Burhinyi doivent être envisagées. Il en est de même pour les études ethnobotaniques qui, dans d'autres pays ont générées des informations scientifiques de grande valeur pour l'aménagement et la gestion durable des forêts (Adomou et al., 2017 ; Hama et al., 2019).

Plusieurs mesures stratégiques pour la conservation des oiseaux et l'ensemble de la biodiversité de la forêt de Burhinyi ont été formulées par les enquêtés, notamment l'abandon de la chasse, l'arrêt de la déforestation, la sensibilisation de la population et la conversion de cette forêt en aire protégée. Nous pensons que l'organisation des séances de sensibilisation de la population dans ce milieu peut réduire tant soit peu la pression actuelle sur l'avifaune. Nous avons enfin constaté que l'initiative de gestion communautaire de la forêt de Burhinyi, envisagée par chefs coutumiers et leaders locaux n'est pas connue par les populations enquêtées. D'où la nécessité de l'organisation des séances des formations, sensibilisations et conscientisation de la population riveraine en rapport avec la conservation des oiseaux et son habitat.

La sensibilisation sur les problèmes environnementaux stimule le sens de la responsabilité individuelle et collective des citoyens face à leur environnement, et contribue à conduire les individus à mieux 
connaître et gérer efficacement les problèmes environnementaux (Kassoum, 2007).

\section{Conclusion}

Les connaissances locales ainsi que l'impact de certaines pratiques au sein de la population riveraine de la forêt de Burhinyi ont été évalués dans six groupements. Les résultats ont relevé 9 activités anthropiques qui pourraient avoir un impact négatif sur l'avifaune. Il a été retenu de ces résultats que dans tous les groupements prospectés, les oiseaux sont couramment abattus pour la consommation. Quatre techniques de chasse d'oiseaux (Catapulte, Pièges artisanaux, Filets japonais et la glue artisanale) ont été inventoriées dans la zone d'étude. Deux techniques de chasse (pièges artisanaux et filets) sont plus utilisées à Cirere, Nirinja et Kalambo. Etant donné l'impact de ces engins de chasse sur l'avifaune, les activités de conservation de l'avifaune, notamment la sensibilisation devraient se concentrer dans trois groupements. La quantité d'oiseaux abattus journalièrement par les chasseurs varie d'un groupement à l'autre ainsi que les taxons affectés. En rapport avec les connaissances ethno-ornithologiques, bon nombre de paysans disposent des connaissances importantes sur les oiseaux dont ils ne souhaiteraient pas la disparition totale. Cette étude a ainsi généré des informations utiles qui pourraient éclairer l'opinion pour une prise de décision en faveur de la conservation communautaire de la biodiversité de la forêt de Burhinyi. Néanmoins, certains thèmes pourraient encore être approfondis comme l'estimation économique des activités anthropiques, les mesures alternatives ainsi que l'impact socioéconomique de la conservation sur la population locale.

\section{CONFLIT D'INTERETS}

Les auteurs confirment qu'ils n'ont aucun conflit d'intérêts à déclarer.

\section{CONTRIBUTIONS DES AUTEURS}

Bertin Murhabale a supervisé la collecte des données sur terrain, l'analyse des données et préparé le manuscrit. Blaise Irenge et Gabriel Biringanine ont participé aux expéditions sur terrain. Frank Bapeamoni a contribué dans l'analyse des données, dans la revue du manuscrit. Charles Kahindo et Dieudonné Upoki ont proposé la méthodologie, revu l'analyse des données et supervisé la rédaction du manuscrit.

\section{REMERCIEMENTS}

Nous tenons à remercier vivement toutes les autorités coutumières de la chefferie de Burhinyi plus particulièrement Messieurs Kahumba et Namuleba, respectivement chefs de groupements de Nirinja et de Cirere pour avoir facilité la collecte des données. Nos sincères remerciements vont également au Council on Africa du Field Museum de Chicago pour le soutien financier.

\section{REFERENCES}

Adomou CA, Dassou HG, Houenon GHA, Alladaye A, Yedomonhan H. 2017. Comprendre les besoins en ressources végétales des populations riveraines pour une gestion durable de la forêt Bahazoun au Sud-Bénin (Afrique de l'Ouest). Int. $J$. Biol. Chem. Sci., 11(5): 2040-2057. http://ajol.info/index.php/ijbcs

Barnosky A, Matzke N, Tomiya S, Wagan GOU, Swartz B, Quental BT, Marshall C, McGuire JL, Lindsey EL, Maguire KC, Lersey B, Ferrer EA. 2011. Has the Earth's sixth mass extinction already arrived?. Nature, 471: 51-57. DOI: http://dx.doi.org/10.1038/ nature09678

BirdLife International. 2018. L'Etat des Oiseaux d'Afrique 2017 : Indicateurs de notre environnement en mutation. Nairobi, Kenya : Partenariat africain de BirdLife International : Kenya.

Brochet A, Van Den Bossche W, Jbour S, Ndang'ang'a P, Jones V, Abdou W, Butchart S. 2016. Preliminary assessment of the scope and scale of illegal killing and taking of birds in the Mediterranean. Bird Conservation International, 26(1): 1-28.

DOI: http://dx.doi.org/10.1017/S09592709150 00416 
Brochet A, Van Den Bossche W, Jones V, Arnardottir H, Damoc D, Demko M, Butchart S. 2019. Illegal killing and taking of birds in Europe oustside the Mediterranean: Assessing the scope and scale of a complex issue. Bird Conservation International, 29(1): 1-40. DOI:

http://dx.doi.org/10.1017/S09592709170 00533

Brooks T, Andriamaro L, Gereau R, Hawkin F, Howell K, Knox D, Langhammer P, Lamoreux JF, Lowry II PP, Luke Q, Matiku P, McKnight MW, Msuya C, Mugo R, Rabarizon H, Rakotobe ZL, Randrianasolo H. 2007. Objectifs et priorités pour la conservation des oiseaux et de la biodiversité d'Afrique. Ostrich, 78(2) : $\quad 115-126 . \quad$ DOI : https://doi.org/10.2989/OSTRICH.78.2.1 .81

Clavel J, Julliard R, Devictor V. 2011. Worldwide decline of specialist species: toward a global functional homogenization? Frontiers in Ecology and the Environment, 9: 222-228. DOI: https://doi.org/10.1890/080216

Chapin FS, Zavaleta ES, Eviner VT, Naylor RL, Vitousek PM, Reynolds HL, Hooper DU, Lavorel S, Sala OE, Hobbie SE, Mack MC, Diaz S. 2000. Consequences of changing biodiversity. Nature, 405: 234-242.

Deikumah JP, Kwafo R, Konadu VA. 2017. Land use types influenced avian assemblage structure in a forestagriculture landscape in Ghana. Ecol Evol., 7: 8685-8697. DOI: https://doi.org/10.1002/EcolEvol

Edderai D, Dame M. 2006. A Census of the commercial bushmeat market in Yaoundé, Cameroon. Oryx, 40: 472-475. DOI:

https://doi.org/10.1017/S0036053060012 56

Fahrig L. 2003. Effects of habitat fragmentation on biodiversity. Annual Review of Ecology Evolution and Systematics, 34: 487-515.
Foley JA, Defries R, Asner GP, Barford C, Bonan G, Carpenter SR, Chapin FS, Coe MT, Daily GC, Gibbs HK, Helkowski JH, Holloway T, Howard EA, Kucharik CJ, Monfreda C, Patz JA, Prentice IC, Ramankutty N, Snyder PK. 2005. Global consequences of land use. Science, 309: 570-574.

Gallai N, Salles JM, Settele J, Vaissiere BE. 2009. Economic valuation of the vulnerability of world agriculture confronted with pollinator decline. Ecological Economics, 68: 810-821.

Gill JA, Norris K, Sutherland WJ. 2001. Why behavioural responses may not reflect the population consequences of human disturbance? Biol. Conserv., 97: 265-268

Hama O, Kamou H, Ali Abdou MM, Saley K. 2019. Connaissances ethnobotaniques et usages de Combretum micranthum dans la pharmacopée traditionnelle au Sudouest de Tahoua (Niger, Afrique de l'Ouest). Int. J. Biol. Chem. Sci., 13(4): 2173-2191.

DOI : http://ajol.info/index.php/ijbcs

Ilboudo A, Soulama S, Hien E, Zombre P. 2020. Perceptions paysannes de la dégradation des ressources naturelles des bas-fonds en zone soudano-sahélienne: cas du sous bassin versant du NakanbéDem au Burkina Faso. Int. J. Biol. Chem. Sci., 14(3): 883-895. DOI : http://ajol.info/index.php/ijbcs

Kassoum T. 2007. De la Sensibilisation des Populations à la Gestion de l'Environnement Urbain dans les quartiers précaires de la ville d'Abidjan. African Population Studies, 22(2): 153 173. DOI: https://doi.org/10.11564/22-2333

Kujirakwinja D, Plumptre A, Twendilonge A, Mitamba G, Mubalama L, Wasso J, Tshombe R. 2019. Etablishing the Itombwe Natural Reserve: Science, participatory, consultations and zoning. Oryx, 53(1): 49-57. DOI: https://doi.org/10.1017/S0030605317001 478

Ladle RJ, Whittaker RJ. 2011.Conservation Biogeography. Wiley-Blackwell: Oxford. 
Lougbégnon OT, Libois RM. 2011. Oiseaux, Birds. In Protection de la nature en Afrique de l'Ouest : une liste rouge pour le Bénin, Neuenschwander P, Sinsin B, Goergen $\mathrm{G}$ (eds). International Institute of Tropical Agriculture: Ibadan, Nigeria ; 204-228.

Lougbegnon OT. 2016. Ecologie et connaissances Ethnozoologiques des quelques espèces d'oiseaux menacés des écosystèmes du Sud du Benin. PhD thesis. Fac Sci, UL, Belgique, p. 146.

Mbete RA, Banga-Mboko H, Ngokaka C, Bouckacka QF, Nganga I, Hornick JL, Vermeulen C. 2011. Profil des vendeurs de viande de chasse et évaluation de la biomasse commercialisée dans les marchés municipaux de Brazzaville, Congo. Tropical Conservation Science, 4(2) : 203-217. DOI: https://doi.org/10.1177/19400829110040 0208

MECNT. 2014. Cinquième rapport national sur la mise en œuvre de la Convention sur la Diversité Biologique. Kinshasa, RDC.

Munay PL. 2004. Habitat loss, resource specialization, and extinction on coral reefs. Global Change Biol., 10 : 16421647.

Nangalire N, Mushagalusa M, Ntamwira N. 2017. Contribution à l'étude de l'abondance et de la diversité des espèces ligneuses de la forêt d'altitude de Burhinyi à l'Est de la République Démocratique du Congo. Geo-Eco-Trop, 41(1): 1-12.

Ndang'ang'a PK, Njoroge JB, Vickery J. 2013. Quantifying the contribution of birds to the control of arthropod pests on kale, Brassica oleracea acephala, a key crop in East African highland farmland. International Journal of Pest Management, 59 (3) : 211-216.

Ntamwira N, Nyakabwa M. 2008. Contribution à l'étude des groupements végétaux postculturaux Burhinyi (Sud-Kivu, RD.Congo) :L'Association à Virectaria major (Virectarietum majoris Habiyaremye 1997. Annales des Sciences, Université Officielle de Bukavu, 1(1) : 54-62.

Olden JD, Poif NL, Douglas MR, Douglas ME, Fausch KD. 2004. Ecological and evolutionary consequences of biotic homogenization. Trends in Ecology and Evolution, 19: 18-24.

Plumptre A, Davenport T, Behangana M, Kityo R, Eilu G, Ssegawa P, Ewango C, Meirte D, Kahindo C, Herremans M, Peterhans J, Pilgrim J, Wilson M, Languy M, Moyer D. 2007. The biodiversity of the Albertine Rift. Biological Conservation, 134: 178194.

DOI: http://dx.doi.org/10.1016/j.biocon.2006.0 8.021

Sandjong Sani RC, Ntoupka M, Ibrahima A, Vroumsia T. 2013. Essai d'analyses de la conception paysanne de l'évolution, de la gestion et de l'utilité du Parc National de Mozogo-Gokoro (Cameroun) en vue de son aménagement. Int. J. Biol. Chem. Sci., 7(6): 2490-2503. http://ajol.info/index.php/ijbcs

Tamisier A, Bechet A, Jarry G, Leufeuvre JC, Le Maho Y. 2003. Effets du dérangement par la chasse sur les oiseaux d'eaux. Rev. Ecol. (Terre vie), 58: 435 - 449.

Tilman D, Fargione J, Wolff B, D'antonio C, Dobson A, Howarth R, Schindler D, Schlesinger WH, Simberloff D, Swackhamer D. 2001. Forecasting agriculturally driven global environmental change. Science, 292: 281284.

Tscharntke T, Klein AM, Kruess A, SteffanDewenter I, Thies C. 2005. Landscape perspectives on agricultural intensification and biodiversity ecosystem service management. Ecology Letters, 8: 857-874.

West P, Igoe J, Brockington D. 2006. Parks and people: the social impact of protected areas. Annual Review of Anthropology, 35: 251-277. 